\title{
"Estándares e indicadores para evaluar la atención de enfermería en unidades psiquiátricas"
}

\author{
"standard and indicators to evaluate \\ the attention of nursing in psychiatric units." \\ M en E.A.S.E. Rafael Antonio Estévez Ramos* ${ }^{*}$ M en A.S.S. Ma. Dolores Martínez Garduño** \\ M en E.A.S.E. Cristina Salvador Martínez ${ }^{\star \star}$, Dra. En Enf. Beatríz Arana Gómez ${ }^{\star \star}$ \\ *Secretaría de Salud, Supervisor de los Servicios de Enfermería del Hospital de Psiquiatría \\ "Dr. Samuel Ramírez Moreno". Instituto de Salud del Estado de México. \\ * Universidad Autónoma del Estado de México, Facultad de Enfermería y Obstetricia, \\ Profesor de Tiempo Completo.
}

\section{Resumen}

El estándar es una declaración explícita de calidad, para determinar su grado de cumplimiento es necesario contar con indicadores, determinando el margen entre el desempeño ideal y el real en los procesos y los resultados de satisfacción de los usuarios. La medición de la calidad de la atención de enfermería es tan importante como extensa, las unidades psiquiátricas no pueden estar ajenas a esto; siendo que la salud mental es factor importante para el desarrollo progresivo y armónico de la sociedad, la inexistencia de estándares e indicadores atenta contra la salud física del paciente, el equilibrio emocional del trabajador, la economía familiar del enfermo y los gastos de la institución; en este sentido se validaron instrumentos con enfoque de

The standard is an explicit declaration of quality, to determine its execution degree it is necessary to have indicators, determining the margin between the ideal acting and the real one in the processes and the results of the users' satisfaction. The mensuration of the quality of the nursing attention is as important as extensive, the psychiatric units cannot be unaware to this; being that the mental health is important factor for the progressive and harmonic development of the society, the nonexistence of standard and indicators attempt against the patient's physical health, the worker's emotional balance, the sick person family economy and the expenses of the institution; in this sense instruments were validated with focus sistema, que se sirvan de guía para evaluar la atención de enfermería en unidades psiquiátricas, a través de procedimientos específicos de enfermería: baño de regadera, alimentación y ministración de medicamentos por vía oral y descanso y sueño; utilizando la Técnica Delphi y Alpha de Cronbach, demostrando que éstos permitirán planear acciones eficientes y eficaces que mejoren la atención de enfermería en psiquiatría, luego que obtuvieron la confiabilidad necesaria para ser utilizados a nivel nacional y ser referentes a nivel internacional.

Palabras claves: Estándares, indicadores, sueño, enfermería, salud mental.

\section{Abstract}

of system that you/they are served as guide to evaluate the nursing attention in psychiatric units, through specific procedures of nursing: watering-can bathroom, feeding and ministración of medications for by oral route and I rest and dream; using the Technical Delphi and Alpha of Cronbach, demonstrating that these will allow efficient and effective actions that improve the nursing attention in psychiatry, to drift then that they obtained the necessary dependability to be used at national level and to be relating at international level.

Key words: Standards, indicators, dream, nursing, mental health. 


\section{INTRODUCCIÓN}

La salud mental es el estado que permite el desarrollo óptimo de cada individuo en el orden físico, intelectual y afectivo, reflejándose en la relación con otros individuos y la capacidad de enfrentar las situaciones de la vida cotidiana.

Se manifiesta por un equilibrio dinámico de la personalidad, la integración, siempre renovada a tendencias contradictorias que permiten establecer relaciones significativas entre el individuo y el mundo, que a la vez preserva la identidad del yo y favorece la adaptación al medio. La salud mental, aporta nuevos elementos para la reflexión en la medida que introduce las variables de las posibilidades al nivel individual y/o social. La salud mental, lejos de ser la ausencia de enfermedades y problemas mentales y psíquicos, es por el contrario la percepción y conciencia de ellos y la posibilidad personal y/o colectiva de tratar de solucionarlos, de modificarlos e intervenir sobre ellos ${ }^{1}$.

Las crecientes necesidades de la población demandan servicios de salud con calidad, dentro de los cinco ejes que marca el Plan Nacional de Desarrollo 2007-2012, en el número 3 encontramos la igualdad de oportunidades, donde su aspecto 3.2 es dedicado a la salud, y dentro de sus estrategias está el ofrecer a toda la población servicios con alta calidad y calidez, para ello es necesario crear un sistema de medición y de esta forma cubrir todos los aspectos que determinan el concepto de calidad, sin restarle importancia a la salud mental, por lo cual se debe de tener en cuenta lo que se entiende por calidad de los servicios que brinda el sector salud ${ }^{2}$

El concepto de calidad está ligado fuertemente a nuestra cultura, por lo que es difícil de definirlo pues en él están presentes una serie de factores y fases impregnados de juicio de valor de qué es lo que constituye para cada uno la calidad ${ }^{3}$.

La calidad como una propiedad que se adquiere de relación de los productos y servicios con el cliente o en el caso de los servicios de salud con el usuario, por lo que para su existencia y valoración se requiere de estos dos elementos: productos (servicio) y usuarios ${ }^{4}$.

La calidad en Enfermería al igual que en otras disciplinas está apegada a la asistencia en concordancia con los avances de la ciencia, con el objetivo de asegurar la continuidad de la misma y la satisfacción del usuario. Para su medición en los servicios de salud es necesario elaborar estándares e indicadores que guíen y orienten la práctica profesional $^{5}$.
Los cambios de hoy se ven reflejados en los movimientos por la calidad, apreciándose transformaciones en los supuestos y conceptos básicos a partir de los que parten los esfuerzos por alcanzar la calidad. Expresando lo anterior a través de cinco etapas: inspección, control estadístico de la calidad, aseguramiento de la calidad, administración de la calidad total, reestructuración de la organización y mejora de procesos"6. En este aspecto se integra además de la comunicación, los medios avanzados que el hombre utiliza para obtener mejores resultados, como es, la computación, el procesamiento de datos y su transformación en información útil, que llevan a mejorar la calidad, la productividad y la obtención de una ventaja competitiva, sobre todo cuando la tecnología es utilizada para un mejor servicio a los clientes, para hacer más sencillo los procesos. Resultando esencial para la calidad debido a que a través de ella se pueden procesar altos volúmenes de información en un mínimo de tiempo y a las necesidades siempre crecientes de los clientes ${ }^{7}$.

En la mejora continua de la calidad de la atención de enfermería se hace necesario la implementación de instrumentos que guíen la práctica y marquen las líneas a seguir para la acción, tanto los estándares como los indicadores son instrumentos esenciales para medir la calidad, pues el estándar es un componente subjetivo que mide y señala el límite diferenciador entre lo que es o no aceptable, mientras que el indicador mide fenómenos específicos, y en su aplicación muestran la tendencia y/o desviación de una actividad ${ }^{5}$, puede emplearse para determinar el grado de cumplimiento de los estándares, determinando el margen que existe entre el desempeño ideal y el real en los procesos clínicos, administrativos y los resultados de satisfacción de los usuarios. Ellos traducen un enunciado cualitativo del estándar en uno cuantitativo (recuentos), promedios, porcentajes o proporción ${ }^{8}$. Estos permiten evaluar la calidad de los procesos y el grado de satisfacción de los usuarios. Así como contar con una guía para la elaboración de los procedimientos, de manera que previenen o evitan desviaciones en los mismos.

De ahí que la responsabilidad de enfermería trasciende los aspectos físicos de los cuidados para implicarse en los propios sentimientos que el paciente tiene respecto a sí, respecto a sus allegados y respecto a sus relaciones sociales, así como la respuesta que el paciente ha de dar al ambiente que lo rodea.

Retomando y analizando diversos elementos de la práctica de enfermería se presenta un concepto propio. "En- 
fermería es la ciencia que se encarga del estudio de las necesidades del individuo y su satisfacción, así como de las respuestas humanas de las personas, generadas en la interacción de estas con su medio ambiente, en su práctica profesional se responsabiliza de la atención de los individuos, familia, grupos y comunidad sanos o enfermos, tiene carácter integrador, pues vincula en su actuar los aspectos físicos, psicológicos, sociales y espirituales de los seres humanos. Sus funciones están dirigidas a la prevención, diagnóstico oportuno, tratamiento y rehabilitación del individuo, con intervenciones independientes y de colaboración con otros profesionales".

Así mismo la enfermería en salud mental y psiquiatría, según Travelbee Joyce, "es un proceso interpersonal por el cual la enfermera presta asistencia al individuo, familia y comunidad, para promover la salud mental, prevenir la enfermedad y afrontar las experiencias de estrés y de enfermedad mental y les ayuda a readaptarse y encontrar significado en estas experiencias" 1 .

Por lo que basado en la experiencia se puede decir que: "La Enfermería Psiquiátrica es una rama de la Ciencia de la Enfermería que se ocupa del estudio y atención de los individuos con alteraciones de la sensopercepción, la conducta y las emociones, caracterizada por mantener una actitud humanista y sociabilizadora, basada no solamente en el cuidado al enfermo mental sino creadora de acciones capaces de favorecer la salud mental de la comunidad y prevenir alteraciones psíquicas en el individuo; quien la ejerce deberá de reunir un conjunto de cualidades y características que de acuerdo a los conocimientos, habilidades, aptitudes y valores le permitan asumir las responsabilidades y competencias requeridas en su desempeño".

A pesar de los avances en la creación e implementación de programas de mejora continua de la calidad en el sector salud en México; las unidades de Atención Psiquiátrica no han sido integradas de manera activa en los mismos, mucho menos la medición de la calidad de los servicios que otorga el personal de enfermería a pacientes adultos con padecimientos psiquiátricos, los cuales reúnen características muy peculiares y de mayor complejidad para su manejo y su integración a roles sociales comunes en otros tipos de padecimientos.

Las consecuencias en perjuicio del usuario pueden ser múltiples cuando no se tienen las precauciones necesarias y las condiciones óptimas para asistir al paciente durante la realización de diferentes procedimientos específicos, como son la ministración de medicamentos por vía oral, descanso y sueño, alimentación por vía oral, el baño de regadera, en este último, cuando éste es susceptible (por diferentes factores) a sufrir accidentes; en los Hospitales Psiquiátricos se ha observado con frecuencia la caída de usuarios durante el baño, quemaduras y otras lesiones ajenas a su padecimiento.

Sin embargo, no existen estadísticas institucionales, ni nacionales al respecto, de igual manera el hecho de que el usuario no ingiera el medicamento indicado, no se le ofrece la correcta alimentación, la vigilancia y control de los factores que pueden llevarlo a una complicación, o no logra un descanso y sueño adecuado, repercutiéndose sobre todo en este tipo de paciente en su recuperación y pone en riesgo su integridad.

Lo anterior son asuntos que la Organización Mundial de la Salud y sus países miembros consideran como problemas que requieren del establecimiento de acciones que permitan prevenir, controlar y disminuir sus efectos. Por otro lado, se ha observado que no se cuenta con instrumentos técnicos-metodológicos que sirvan de guía para la unificación de criterios de supervisión operativa del cuidado de enfermería y la sistematización de sus intervenciones, por lo que sí no se cuenta con ellos no será posible avanzar en la estandarización y unificación de criterios y el establecimiento de indicadores que puedan medir la calidad de la atención de enfermería a usuarios con afecciones psiquiátricas ${ }^{9}$ y 10

\section{METODOLOGÍA}

El presente trabajo es el reporte de un estudio de tipo descriptivo, transversal, y exploratorio, pertenece a la línea de administración en los servicios de enfermería.

Para la construcción del marco referencial, se realizó revisión bibliográfica y documental.

\section{La metodología se desarrollo en cuatro etapas:}

- 1ra. Elaboración del instrumento y selección de expertos.

- 2da. Envío del instrumento a expertos y análisis de observaciones.

- 3ra. Envío de instrumento modificado y validación por parte de los expertos.

- 4ta. Determinación de confiabilidad estadística.

Como instrumentos se utilizaron cuestionarios, elaborados con enfoque sistémico (proceso, estructura y resultado), tomando a Donabedian como referencia y siguiendo 
las guías la Comisión Interinstitucional de Enfermería y del Instituto Nacional de Cardiología "Dr. Ignacio Chávez"; además de los conocimientos teóricos y la práctica de manera directa con los usuarios con afecciones psiquiátricas ${ }^{3}$.

Para la realización de este trabajo se utilizó la Técnica Delphi o Técnica de Expertos contando con la participación de 25 expertos de Instituciones de Atención Psiquiátrica al adulto, de la Secretaría de Salud de los Estados Unidos Mexicanos. Para la selección de los mismos se tomó como base la definición que da al respecto la teórica de enfermería Patricia Benner en su artículo "Del principiante al experto: excelencia y poder de la enfermería clínica".

En la determinación de la muestra para la aplicación de los instrumentos como fase final (4ta. Etapa), se utilizó el paquete estadístico Epi info 2000, con un 95\% de confiabilidad, obteniéndose una muestra de 14 enfermeras a evaluar por cada procedimiento.

\section{criterios de inclusión:}

- Personal que esté directamente involucrado con alguna de los procedimientos a evaluar.

- Personal que no se encuentre disfrutando de alguna prestación (económicos, vacaciones, etc.)

- Personal que no se encuentre en formación.

\section{Criterios de exclusión:}

- Personal que no esté directamente involucrado con alguna de los procedimientos a evaluar.

- Personal que se encuentre disfrutando de alguna prestación (económicos, vacaciones, etc.)

- Personal en formación (estudiantes y pasantes).

- Personal de enfermería que no desee participar.

- Personal de enfermería que no cumpla con los criterios de inclusión.

\section{Criterios de eliminación:}

- Todo instrumento que carezca de algún dato.

\section{Metodo:}

Inductivo - Deductivo.

\section{Límites de la investigación:}

Tiempo: De Febrero del 2007 a Junio del 2008.

Espacio: 17 Entidades Federativas de los Estados Unidos Mexicanos: Distrito Federal, Estado de México,
Aguascalientes, Baja California Sur, Campeche, Colima, Chihuahua, Durango, Guanajuato, Jalisco, Michoacán, Oaxaca, Sinaloa, Sonora, Tabasco, Veracruz, Yucatán.

\section{Análisis de datos:}

Los resultados que se obtuvieron se analizaron a través del sistema estadístico SPSS versión 15, aplicando Alpha de Cronbach y presentándolos en cuadros y gráficas.

\section{Presentación de resultados}

La descripción de los resultados se realiza por cada uno de los procedimientos analizados, describiendo como estuvo constituido el instrumento que se les presentó a los expertos, posteriormente el promedio general alcanzado por cada instrumento y finalmente como quedó constituido cada uno de los instrumentos, así como la puntuación alcanzada por alpha de cronbach.

El instrumento de alimentación por vía oral fue elaborado con 20 ítems, 5 de estructura, 10 de proceso y 5 de resultado (Instrumento No.1).

El promedio de aprobación por etapas del instrumento para la evaluación del procedimiento de alimentación por vía oral resultó ser para estructura de 4.32, para el proceso de 4.16 y para resultados de 4.02 en la escala de Likert (Gráfico No.2).

El de ministración de medicamentos por vía oral fue elaborado con 18 ítems, 6 de estructura, 8 de proceso y 4 de resultados (Instrumento No.2).

En relación al promedio de aprobación por etapas para el instrumento de ministración de medicamentos por vía oral, la de proceso obtuvo la mayor puntuación con 4.54, seguida de la de estructura con 4.43 y resultado con 4.24 (Gráfico No.4).

Así mismo para el descanso y sueño se tuvieron en cuenta 15 ítems, 5 de estructura, 6 de proceso y 4 de resultados (Instrumento No.3).

En el promedio por fases o etapas el comportamiento en cuanto al instrumento de descanso y sueño fue de la siguiente manera, 3.92 para resultados, 4.29 para proceso y 4.59 para estructura (Gráfico No.6).

El instrumento para baño de regadera inicialmente contó con 18 ítems de los cuales 6 fueron de estructura, 7 de proceso y 5 de resultados. (Instrumento No.4).

La etapa con mayor puntuación en el instrumento de baño de regadera fue la de proceso con 4.48, en orden descendente se ubica, estructura con 4.41 y resultado con 4.14 (Gráfico No.8). 
En términos generales el promedio de aprobación para alimentación por vía oral fue de 4.17, seguido en orden ascendente por el instrumento de descanso y sueño con 4.27, baño de regaderas con 4.34 y ministración de medicamentos por vía oral con 4.40 (Cuadro No.1).

Finalmente los instrumentos quedaron constituidos de la siguiente forma:

El instrumento de alimentación por vía oral con 25 ítems, 14 de estructura, 6 de proceso y 5 de resultado. El de ministración de medicamentos por vía oral con 20 ítems, 11 de estructura, 7 de proceso y 2 de resultados. Así mismo el instrumento de descanso y sueño cuenta con 17 ítems, 9 de estructura, 5 de proceso y 3 de resultados. El instrumento para baño de regadera quedó constituido por 31 ítems de los cuales 20 son de estructura, 8 de proceso y 3 de resultados.

La confiabilidad obtenida por Alpha de Cronbach quedo de la siguiente manera: Instrumento de baño de regadera .857, ministración de medicamentos por vía oral .788, alimentación por vía oral .779 y descanso y sueño .511

\section{DISCUSIÓN}

La validación de instrumentos de evaluación para procedimientos de enfermería, es hoy en día indispensable para mejorar la calidad del cuidado que se brinda a los usuarios de los servicios profesionales de enfermería.

El área de psiquiatría ha sido poco atendida en este aspecto, muestra de ello es la inexistencia de estándares e indicadores específicos para este tipo de usuario.

De este modo los procedimientos más comunes y por ello estudiados en esta investigación son: Alimentación vía oral, baño de regadera, ministración de medicamentos y por último descanso y sueño.

Inicialmente el instrumento para evaluar el procedimiento de alimentación por vía oral estaba constituido por 20 items, mismo que de acuerdo a las opiniones de los expertos se fue modificando, se eliminaron algunas preguntas las cuales se consideraron repetitivas e innecesarias, se unificaron en criterios más generales las de mayor relevancia o muy importantes según lo opinado por cada experto; a pesar de todo, este instrumento fue el que menos observaciones tuvo, su puntuación menor de acuerdo a la escala de Likert fue de 3, por lo que desde un inicio su contenido se encontraba dentro del rango, pues esta calificación corresponde al criterio de necesario.
En cuanto a la calificación por etapas el instrumento de alimentación por vía oral se ubicó entre muy necesario y totalmente necesario, sin restar importancia una etapa a otra, dado que en cuestiones de evaluación de procesos es importante no sólo el proceso como tal, sino también la estructura con que se cuenta para realizarlo y cómo medimos su resultado, por lo que finalmente el instrumento obtuvo una calificación de muy necesario (4.17). A pesar de esto y atendiendo a las observaciones realizadas por los expertos al instrumento se le realizaron modificaciones, quedando con 25 items, 14 de estructura, 6 de proceso y 5 de resultado, mismos que fueron validados en su 100\% por los 25 expertos que emitieron su aprobación por escrito, reflejándose este resultado en la confiabilidad que se obtuvo de Alpha de Cronbach (.779), por lo que se afirma que el instrumento es aplicable y confiable.

Para elaborar el instrumento de ministración de medicamentos por vía oral se tuvo en cuenta las características tan peculiares de los usuarios a los que va dirigido, con el mismo enfoque de estructura, proceso y resultado lo constituyó 18 items, los cuales fueron sometidos a la opinión de los 25 expertos, obteniendo tres calificaciones de necesario (3, 3.2 y 3.8), el resto lo consideró entre muy necesario y totalmente necesario, igualmente el promedio de aprobación por etapas fue muy alto, ubicándose entre muy necesario y totalmente necesario, al igual que el instrumento anterior, obteniendo su mayor calificación en la parte de estructura, sin restarle importancia a las otras dos pues todas como un sistema que forman, resultaron ser muy necesarias con un promedio general de 4.40, en la escala de Likert.

En la determinación del instrumento final para la ministración de medicamentos por vía oral, se realizaron las modificaciones sugeridas por los expertos y las que a nuestro criterio eran necesarias modificar para que las preguntas fueran más claras y precisas al momento de aplicarse, quedando finalmente el instrumento constituido por 11 items de estructura, 7 de proceso y 2 de resultado, validados y aprobados por los 25 expertos participantes y obteniendo un Alpha de Cronbach, luego de su aplicación de .788, demostrando así su alta confiabilidad.

En el instrumento para medir la atención de enfermería durante el descanso y sueño del usuario con afecciones psiquiátricas fue necesario inicialmente construir un instrumento compuesto por 15 items, 5 de estructura, 6 de proceso y 4 de resultado, los cuales fueron considerados en su mayoría, por parte de los expertos, como muy nece- 
sario, lo que demuestra que el instrumento fue importante para los ellos, mostrando su aprobación también en cada una de las fases o etapas, obteniendo un promedio general de muy necesario; a pesar de lo anterior y siguiendo lo marcado por la técnica utilizada se le realizaron modificaciones teniendo en cuenta las observaciones realizadas por el grupo que colaboró en esta investigación, quedando un instrumento compuesto por 17 items, 9 de estructura, 5 de proceso y 3 de resultado, los cuales fueron validados en su contenido y forma por el $100 \%$ de los expertos, se obtuvo un Alpha de Cronbach de .511, ubicando al instrumento en una confiabilidad media desde el punto de vista estadístico, lo anterior no quiere decir que este instrumento no cubra lo necesario para su aplicación, puesto que desde un inicio fue aprobado por los expertos, sino que tal vez haya que trabajar más en el, lo anterior queda sustentado en la aprobación final que dan los expertos, donde como se menciona anteriormente, el $100 \%$ coincide en que el instrumento se ha validado en cuanto a consistencia.

El cuarto instrumento contemplado para evaluar la atención de enfermería en unidades psiquiátricas fue el correspondiente al procedimiento de baño de regadera, al cual, igual que a los otros tres, se le integraron items con enfoque de sistema, 18 en total, 6 de estructura, 7 de proceso y 5 de resultado, registrándose en este la menor calificación en el rango de necesario, situación que además de considerarse como aprobatoria, no resultó ser muy relevante ya que por etapas o fases (estructura, proceso y resultado), fueron considerados como muy necesario y su promedio general fue de igual resultado. Las observaciones realizadas por los expertos fueron similares al resto de los instrumentos, por lo que con el fin de llegar a congruencias entre los participantes se realizaron las modificaciones que se consideraron pertinentes al instrumento quedando finalmente constituido por 31 items, 20 de estructura, 8 de proceso y 3 de resultado, los cuales fueron aprobados y validados en su contenido y forma por el $100 \%$ de los expertos, obteniendo éste luego de su aplicación la mayor confiabilidad a través de Alpha de Cronbach (.857).

Estos instrumentos cumplen con las características descrita por el Instituto Nacional de Cardiología para la construcción de estándares e indicadores para incidir de manera efectiva en la mejora continua de la calidad de la atención de enfermería, para lo cual se hace necesario la implementación de instrumentos que guíen la prác- tica y marquen las líneas a seguir para la acción, tanto los estándares como los indicadores son instrumentos esenciales para medir la calidad, pues el estándar es un componente subjetivo que mide y señala el límite diferenciador entre lo que es o no aceptable, mientras que el indicador mide fenómenos específicos, y en su aplicación muestran la tendencia y/o desviación de una actividad $^{5}$.

Como se ha descrito anteriormente la calidad total (TQ) es un sistema administrativo enfocado hacia las personas, que busca un incremento continuo en la satisfacción del usuario a un costo real cada vez más bajo, estos instrumentos servirán como facilitadores para lograr la ya mencionada calidad total, donde a partir de la correcta ejecución de los procedimientos antes planteados y su evaluación, se podrán obtener mejores resultados en la satisfacción del usuario, que repercutan además directamente sobre el costo beneficio?.

Hasta el momento en otros instrumentos planteados como indicadores, se percibe que miden únicamente el proceso, o sea cómo el personal de enfermería realiza el procedimiento, en este estudio los estándares e indicadores fueron validados con enfoque de sistema (estructura, proceso y resultados), considerando que, las características estructurales del lugar donde se otorga la atención, influye sobre el proceso de la atención, disminuyendo o aumentando su calidad, así como los cambios en el proceso de dicha atención tendrán gran influencia sobre el estado de salud (resultados), en su definición más amplia ${ }^{11}$

El resultado es otra parte importante de un procedimiento de enfermería, pues éste representa un estado, conducta o percepción del individuo, familia o comunidad, el cual sólo puede ser medido a través de la aplicación de un indicador de resultados, los cuales determinan el estado del usuario en relación al resultado obtenido ${ }^{12}$.

Con el enfoque que se les dio a estos instrumentos se puede afirmar que las instituciones que lo apliquen de manera correcta podrán estar preparadas para la evaluación de la atención tanto interna como externa, ya que su validación fue sometida por expertos de todo el país, donde las condiciones estructurales podrán tener similitudes pero no igualdad, la cultura organizacional depende de la estructura con que cuente los diferentes institutos de salud de cada entidad federativa, los grupos de usuarios que se atienden son diferentes en cuanto a género, cultura, patología, cronicidad o no, etc., lo anterior se afirma 
teniendo en cuenta que la evaluación interna es la que realiza la propia institución con el objetivo de favorecer la satisfacción de los usuarios, crear estándares, compararlos con otras unidades prestadoras de servicios similares; la externa es cuando es sometida a una supervisión por organismos ajenos a la unidad, por expertos en la prestación de esos servicios, a través de herramientas metodológicas y sistemas de evaluación acreditados para validar los servicios que presta la unidad ${ }^{5}$.
Al ser estos procedimientos los que con mayor frecuencia se realizan en las unidades de atención psiquiátrica, pero además en los que mayor incidencias se han registrado, en ocasiones no por la forma en que se realiza el proceso, sino por las condiciones estructurales en que se realiza, se pone en riesgo no sólo la integridad del usuario, sino la del personal de enfermería ejecutante de la acción, es por eso que en el momento que ellos sean atendidos en las unidades donde se apliquen y

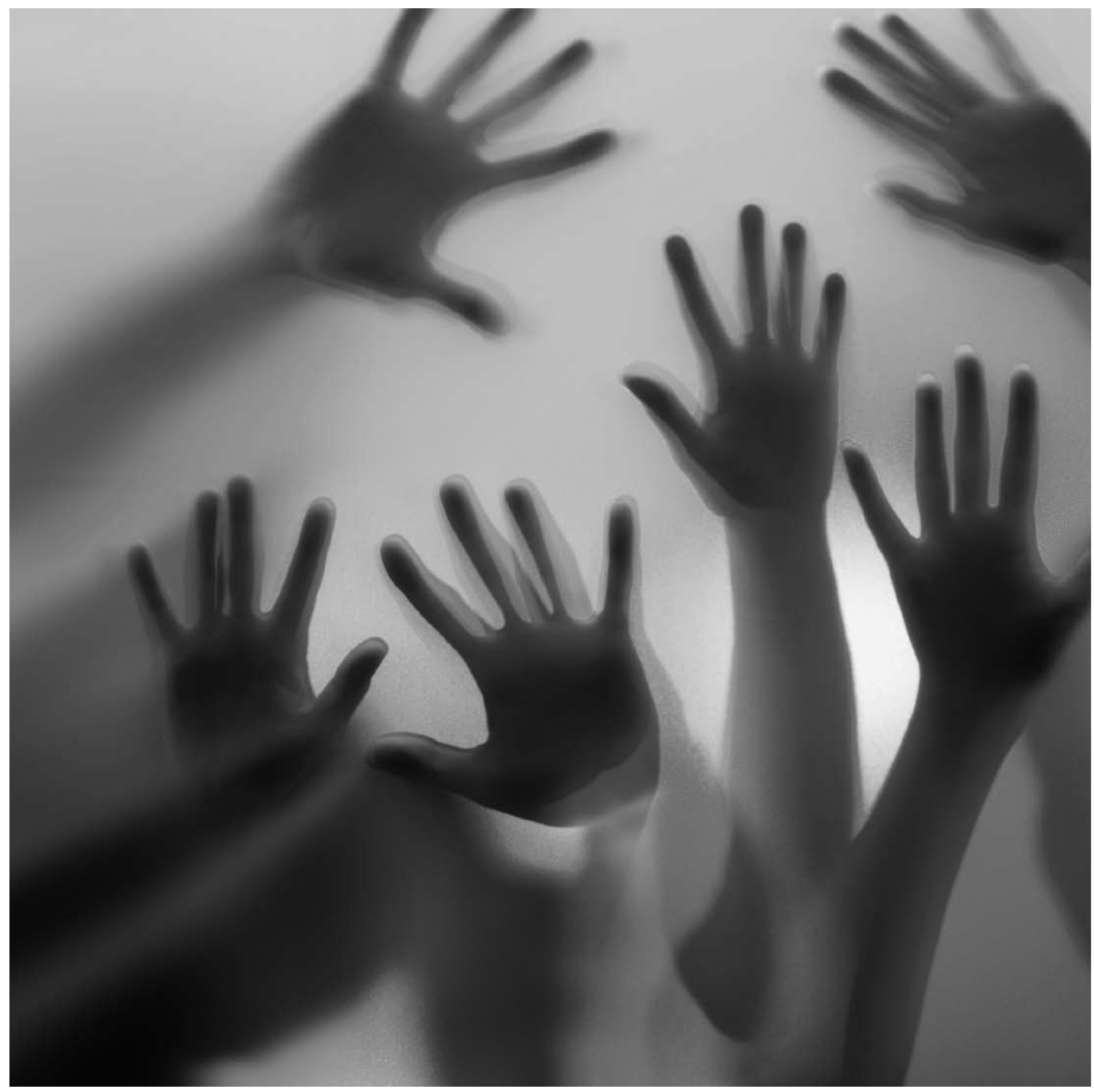


teniendo en cuenta su enfoque de sistema, se podrá lograr una gestión orientada no sólo a las necesidades del usuario, sino también a la de los empleados, la propia institución y la familia, pues se podrán evitar accidentes y así lograr calidad total en la atención que se proporciona, pues se tendrá entonces un control y aseguramiento de la calidad ${ }^{4}$.

También se demuestra que estos instrumentos dan cumplimiento al Programa de Mejora de la Calidad de la Salud Mental, propuesto por la Organización Mundial de la Salud con respecto a Mejora de la Calidad, a través de la revisión de los mecanismos, donde se plantean cinco pasos; Paso 1. Orientar la política hacia la mejora de la calidad, Paso 2. Diseñar un documento de estándares, Paso 3. Establecer procedimientos de acreditación, Paso 4. Monitorizar el servicio de salud mental empleando los mecanismos de calidad, y Paso 5. Integrar la mejora de la calidad en la gestión y prestación de servicios ${ }^{9}$

Los instrumentos validados tienen correspondencia con la misión planteada por la Organización Panamericana de la Salud (OPS) en su Plan Estratégico 2008-2012, "Liderar esfuerzos colaborativos estratégicos entre los Estados Miembros y otros aliados, para promover la equidad en salud, combatir la enfermedad, y mejorar la calidad y prolongar la duración de la vida de los pueblos de las Américas"13

Estos instrumentos responden a algunas de las acciones más importantes de seguridad del paciente planteadas en el foro "Nuevos rumbos de las políticas de salud en México", celebrado el 25 de abril del 2007, donde se refiere a la misión antes mencionada del plan estratégico plateado por la Organización Panamericana de la Salud (OPS), a través de diez acciones para la seguridad del paciente.

1) Manejo de medicamentos (Las 5 " $C$ ").

2) Identificación del paciente.

3) Comunicación clara.

4) Uso de protocolos y/o guías diagnósticas.

5) Cirugías y procedimientos (Las 4 "C").

6) Caídas de pacientes.

7) Infecciones nosocomiales.

8) Factores humanos.

9) Clima de seguridad.

10) Hacer corresponsable al paciente ${ }^{14}$.

Al analizar el formato de análisis (estructura, proceso y resultado) y hacer un comparativo con el proceso en- fermero, se puede observar que la parte de estructura se relaciona con el material y equipo necesario para la realización del procedimiento, el proceso incluye valoración, diagnóstico, planeación y ejecución, y el resultado que es la evaluación de lo ejecutado. Se observa así, que la metodología es similar, lo que garantiza un cuidado de enfermería de excelencia al aplicar las intervenciones propias de la disciplina avaladas por expertos tanto teóricos (formación) como prácticos (experiencia en el área).

\section{CONCLUSIONES Y PROPUESTAS}

Con los cuatro instrumentos planteados inicialmente y luego de llegar a congruencias trascendentales a través de la opinión de los expertos, quedan validados en consistencia, así como por su confiabilidad luego de la aplicación, mismos que serán útiles para la evaluación de la atención de enfermería en unidades de atención psiquiátrica, apoyando así las acciones del Programa Nacional de Salud, y dando cumplimiento a las estrategias planteadas en el plan rector de la Comisión Permanente de Enfermería ${ }^{5}$.

Finalmente se puede afirmar que estos instrumentos pueden ser utilizados plenamente en las unidades psiquiátricas a nivel nacional y servir de referencia a nivel internacional.

Por lo que, por la utilidad de los mismos, estos están en condiciones de poder ser difundidos en las instituciones psiquiátricas con el objetivo de que todo el personal de enfermería conozca los avances en el área y se fomente la cultura de calidad en el personal de enfermería, así como promover el uso de estándares e indicadores para la atención de enfermería, garantizando así la seguridad del paciente y satisfaciendo las necesidades tanto de los usuarios internos como externos.

Se recomienda además que estos indicadores sean revisados plenamente como mínimo cada cuatro años, a fin de mantenerlos actualizados y vigentes para la medición de la calidad, que sean punto de partida para la elaboración de otros estándares e indicadores para otros procedimientos requeridos para la práctica de la enfermería psiquiátrica.

Como parte de la materialización de este estudio se deberá gestionar que estos estándares e indicadores sean valorados por las autoridades competentes, para su aplicación en todas las unidades de atención psiquiátrica del país. 


\section{UNIVERSIDAD AUTÓNOMA DEL ESTADO DE MÉXICO \\ FACULTAD DE ENFERMERÍA Y OBSTETRICIA \\ COORDINACIÓN DE ESTUDIOS DE POSGRADO \\ MAESTRÍA EN ENFERMERÍA \\ INSTRUMENTO DE EVALUACIÓN}

\section{PROCEDIMIENTO: ALIMENTACIÓN POR VÍA ORAL}

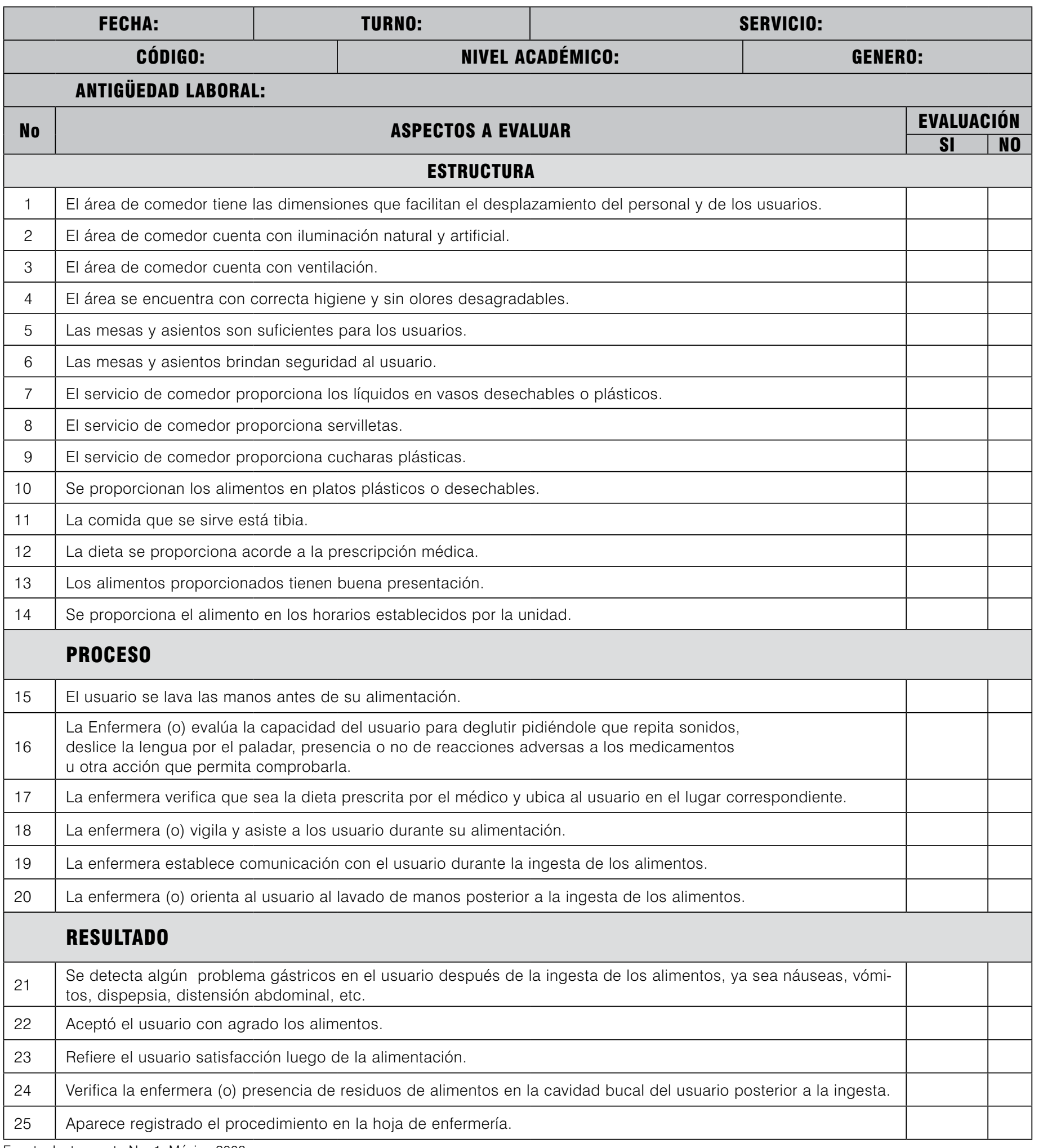

Fuente: Instrumento No. 1, México 2008 


\section{INSTRUMENTOS. MINISTRACIÓN DE MEDICAMENTOS POR VÍA ORAL}

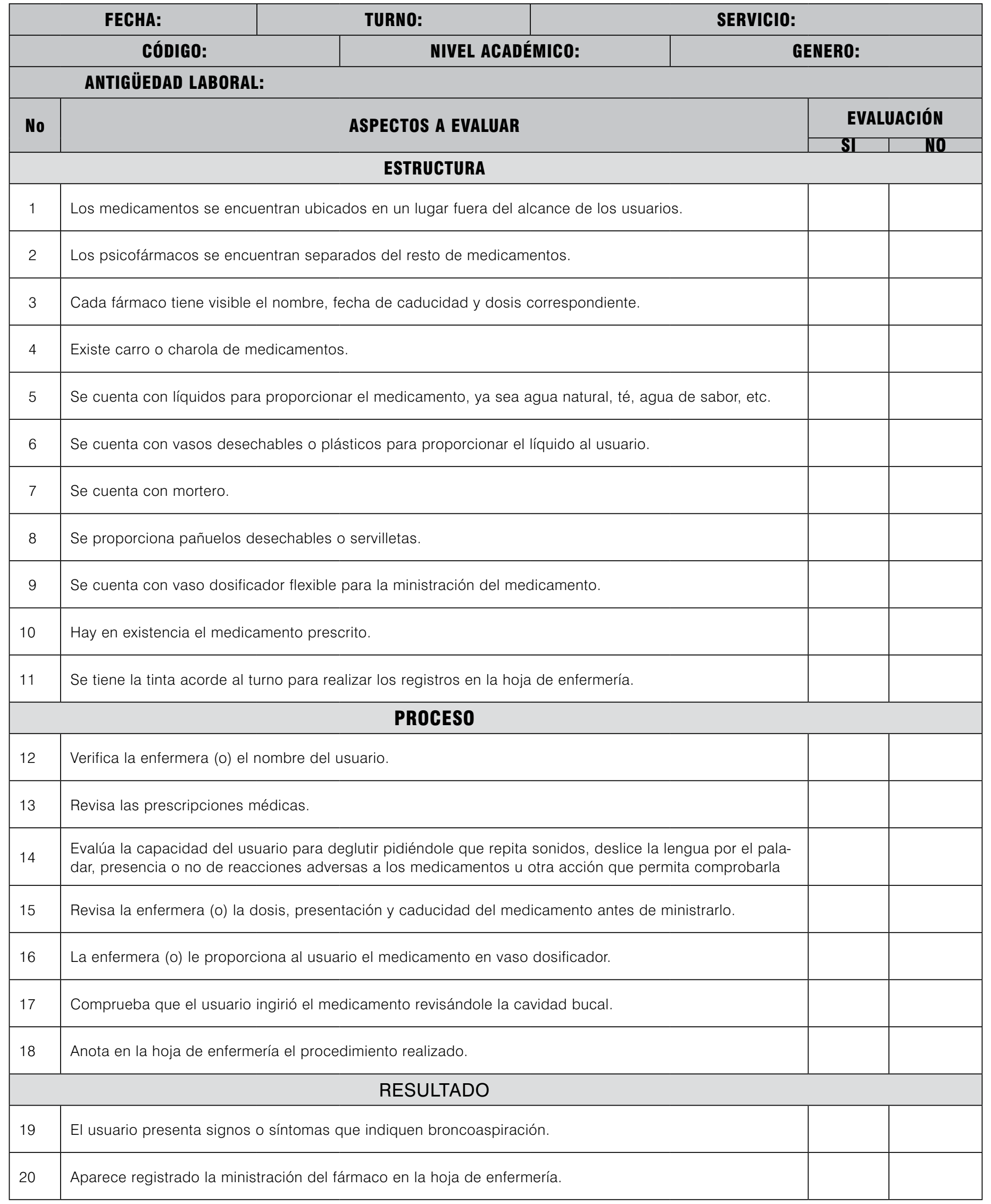

Fuente: Instrumento No. 2, México del 2008. 
INSTRUMENTO. DESCANSO Y SUEÑO

\begin{tabular}{|c|c|c|c|c|c|}
\hline & FECHA: & TURNO: & \multicolumn{3}{|c|}{ SERVICIO: } \\
\hline \multicolumn{3}{|c|}{ CÓDIGO: } & NIVEL ACADÉMICO: & \multicolumn{2}{|c|}{ GENERO: } \\
\hline \multicolumn{6}{|c|}{ ANTIGÜEDAD LABORAL: } \\
\hline \multirow{2}{*}{ No } & \multirow{2}{*}{\multicolumn{3}{|c|}{ ASPECTOS A EVALUAR }} & \multicolumn{2}{|c|}{ EVALUACIÓN } \\
\hline & & & & SI & NO \\
\hline \multicolumn{6}{|c|}{ ESTRUGTURA } \\
\hline 1 & \multicolumn{3}{|c|}{ El espacio de los dormitorios permite el desplazamiento de los usuarios y personal. } & & \\
\hline 2 & \multicolumn{3}{|c|}{ El área de dormitorios se encuentra libre de ruidos. } & & \\
\hline 3 & \multicolumn{3}{|c|}{ El área de dormitorios cuenta con iluminación natural y artificial. } & & \\
\hline 4 & \multicolumn{3}{|c|}{ El área de dormitorios tiene ventilación. } & & \\
\hline 5 & \multicolumn{3}{|c|}{ La cama se encuentra en buen estado. } & & \\
\hline 6 & \multicolumn{3}{|c|}{ El colchón se encuentra en buen estado. } & & \\
\hline 7 & \multicolumn{3}{|c|}{ Se le proporciona sabanas al usuario. } & & \\
\hline 8 & \multicolumn{3}{|c|}{ Se le proporciona cobertor al usuario. } & & \\
\hline 9 & \multicolumn{3}{|c|}{ La ubicación del área de enfermería permite la vigilancia estrecha del usuario. } & & \\
\hline \multicolumn{6}{|c|}{ PROCESO } \\
\hline 10 & \multicolumn{3}{|c|}{ La enfermera (o) verifica que el usuario se acueste en su cama. } & & \\
\hline 11 & \multicolumn{3}{|c|}{ La enfermera (o) valora la presencia de insomnio en el usuario. } & & \\
\hline 12 & \multicolumn{3}{|c|}{$\begin{array}{l}\text { Se reportan actividades con el usuario durante el día que promuevan y lo induzcan al descanso } \\
\text { y sueño. }\end{array}$} & & \\
\hline 13 & \multicolumn{3}{|c|}{ Realiza la enfermera (o) recorridos continuos por el servicio. } & & \\
\hline 14 & \multicolumn{3}{|c|}{ Se interrumpe el descanso o sueño del usuario por razones ajenas a su tratamiento. } & & \\
\hline \multicolumn{6}{|c|}{ RESULTADO } \\
\hline 15 & \multicolumn{3}{|c|}{ El usuario logra dormir de 6 a 8 horas nocturnas. } & & \\
\hline 16 & \multicolumn{3}{|c|}{$\begin{array}{l}\text { Al despertar el usuario, se manifiestan en él signos de insomnio como: cefalea, abotamiento, astenia, } \\
\text { mal carácter, etc. }\end{array}$} & & \\
\hline 17 & \multicolumn{3}{|c|}{ La enfermera (o) registra en la hoja de enfermería la evolución del descanso y sueño del usuario. } & & \\
\hline
\end{tabular}

Fuente: Instrumento No. 3, México 2008 
PROCEDIMIENTO: BAÑo DE REGADERA

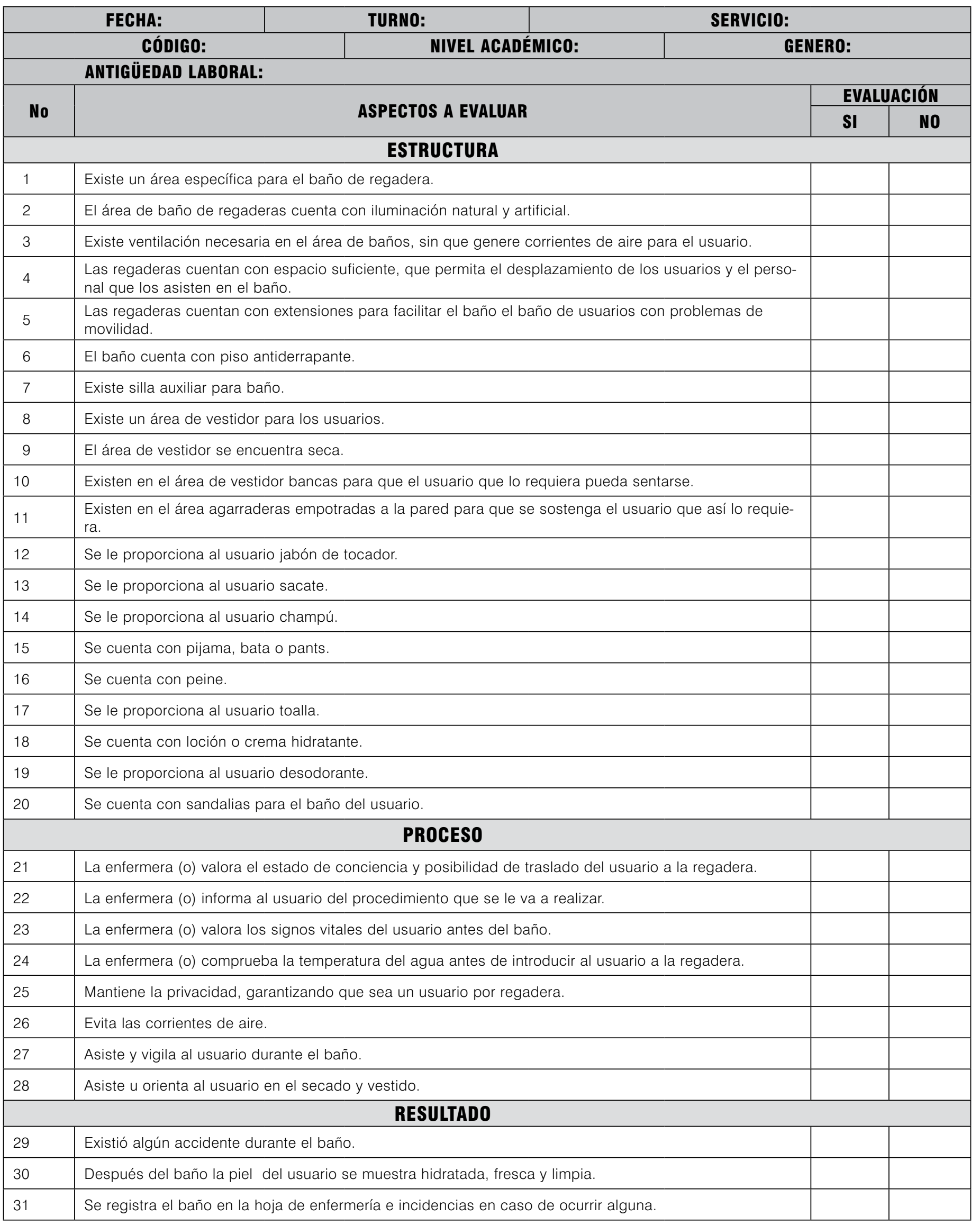

Fuente: Instrumento No. 4, México 2008. 


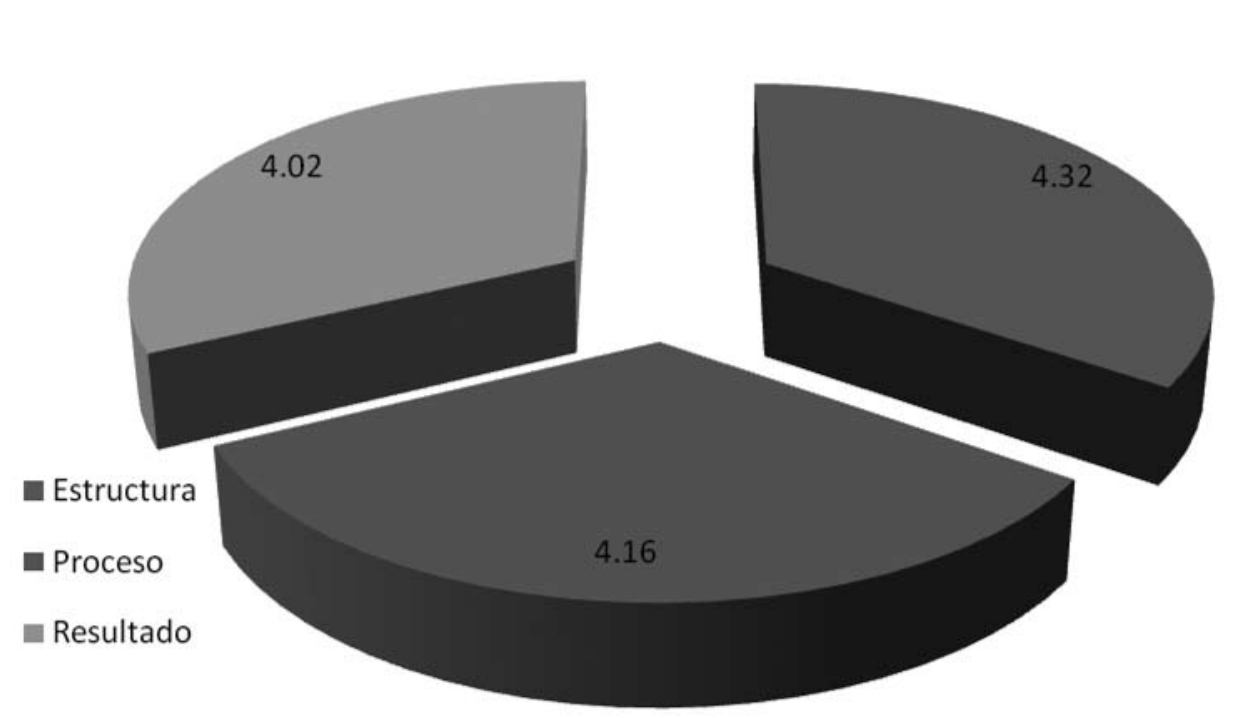

GRÁFICO No. 2

RANGO DE APROBACIÓN POR ÉTAPAS ALIMENTACIÓN POR VÍA ORAL

Fuente: Instrumento aplicado, México 2007.

GRÁFICO No. 4

RANGO DE APROBACIÓN POR FASES EN MISTRACIÓN DE MEDICAMENTOS POR VÍA ORAL

Fuente: Instrumento aplicado, México 2007.


GRÁFICO No. 6

RANGO DE APROBACIÓN POR FASES DESCANSO Y SUEÑO

Fuente: Instrumento aplicado, México 2007 


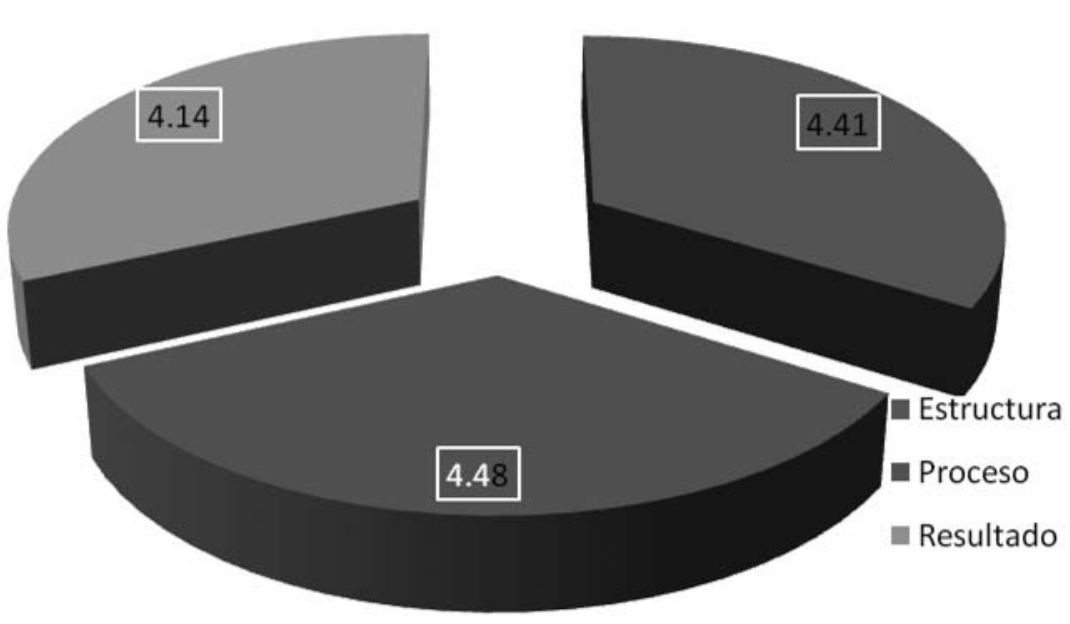

CUADRO No. 1

PROMEDIO DE APROBACIÓN DE INSTRUMENTOS POR EXPERTOS FASE INICIAL

PROCEDIMIENTO

ALIMENTACIÓN POR VÍA ORAL

MINISTRACIÓN DE MEDICAMENTOS POR VÍA ORAL

DESCANSO Y SUEÑO

BAÑO DE REGADERAS

Fuente: Instrumento aplicado, México 2007.

CUADRO No. 3

CONFIABILIDAD POR ALPHA DE CROWMBACH

\section{PROCEDIMIENTO}

BAÑO DE REGADERA.

ALIMENTACIÓN POR VÍA ORAL.

MINISTRACIÓN DE MEDICAMENTOS POR VÍA ORAL.

DESCANSO Y SUEÑO.

.655
GRÁFICO No. 8

RANGO DE APROBACIÓN

POR FASES BAÑO DE REGADERA

Fuente: Instrumento aplicado, México 2007.
PROMEDIO DE APROBACIÓN

4.17

4.40

4.27

4.34
779

.788

CONFIABILIDAD

857 


\section{REFERENCIAS BIBLIOGRÁFICAS}

1 Megías - Lizcano, Francisco - Serrano Parra, María Dolores. Enfermería en psiquiatría y salud Mental. 2da. Edición. España. Edit. Editor Difusión Avances de Enfermería (DAE, S.L.). 2002. p. 276 - 286.

2 Plan Nacional de Desarrollo 2007 - 2012, (consultado el 22/09/2007). Disponible en http://pnd.presidencia.gob. $\mathrm{mx} /$.

3 Lemus Calderón, Jorge Dr. - Aragües y Oroz, Valentín Dr. Auditoria médica y profesional integral. La calidad de la atención en los servicios de salud. Argentina. Edit. Corpus Editorial. 2006. 15,32 pp.

4 Alvarez Heredia, Francisco. Calidad y auditoria en salud. Colombia. Edit. Ecoe Ediciones. 2003. p. 15 - 156.

5 Ortega, Ma. Carolina. Suárez, María Guadalupe. Manual de evaluación del servicio de calidad en enfermería. México. Edit. Médica Panamericana. 2006. P. 1 - 12.

6 Gutiérrez Pulido, Humberto. Calidad total y productividad. $2^{a}$. Edición. México. Edit. McGraw-Hill Interamericana. 2006. 10 pp.

7 James R. Evans. Administración y control de la calidad. México. Edit. Internacional Thompson Editores. 2005. p. $17-62$.

8 Ministerios de Salud Pública de Honduras - Ecuador - Nicaragua. (2003) Manual de medición de la calidad, estándares e indicadores. Proyecto colaborativo interpaíses. (consultado el 03/05/2007). Disponible en http:/wwwqaproject.org/pubs/PDFs/LACHSR/Anexes/Anexo\%202. Manual\%20de20\%Capacitaci\%C3\%B3n.Unidad/20Dos. pdf.

9 Organización Mundial de la Salud. Conjunto de guías sobre servicios y políticas de salud mental. España. Edit. Editores Médicos, S.A. EDIMSA. 2003. p. 14,24.

10 Comisión Interinstitucional de Enfermería. Evaluación de la calidad de los servicios de enfermería; Tres nuevos indicadores para la prevención de infecciones y seguridad del paciente. Subsecretaría de Innovación y Calidad. México. 2006. p. 1 - 3.

11 Donabedian, Avedís. La calidad de la atención médica. México. Edit. Ediciones científicas La Prensa Médica Mexicana. 1984. p. 98.

12 Moorhead, Sue. Johnson, Marion. Maas Meridean. Clasificación de resultados de enfermería (Noc). España. Edit. Mosby. 2005. p. XXI.

13 Organización Panamericana de la Salud. Plan Estratégico 2008-2012. (consultado el 22/05/2008). Disponible en http://www.paho.org/Spanish/GOV/CSP/od328-intro-s.pdf.
14 Cámara de diputados. Seguridad del paciente en la atención médica "Foro nuevos rumbos de las políticas de salud en México". (consultado el 22/05/2008). Disponible en www.salud.gob.mx.

15 Balderas Pedrero, Ma. De la Luz. Administración de los servicios de enfermería. México. Edit. McGraw-Hill Interamericana. 2006. $160 \mathrm{pp}$.

16 Basset Machado, Ihosvany. Estévez Ramos, Rafael A. Rodríguez Díaz, Miriam. Manual de organización del departamento de enfermería. Hospital Psiquiátrico "Dr. Samuel Ramírez Moreno". México. 2007. p. 7 - 40.

17 Cárdenas Becerril, Lucila. La profesionalización de la enfermería en México. Un análisis desde la sociología de las profesiones. España. Edit. Ediciones Pomares. 2005. $11 \mathrm{pp}$.

18 Carpenito, Lynda Juall. Manual de diagnósticos de enfermería. 9na. Edición. España. Edit. Mc Graw - Hill Interamericana. 2002. p. 3 -14.

19 Díez Domingo, Ma. Isabel y col. de aut. Manual de enfermería. España. Edit. Lexus editores. 2003. 20 pp.

20 Gallar, Manuel. El paciente con trastornos mentales, en ciencias de la salud. Promoción de la salud y apoyo psicológico al paciente. 3ra. Edición. España. Edit. Thompson. 2002. 41 pp.

21 Gordon, Marjory. Manual de diagnósticos enfermeros. 10ma. Edición. España. Edit. Mosby. 2003. 352 pp.

22 Gutiérrez Pulido, Humberto. Calidad total y productividad. 2da. Edición. México. Edit. Mc Graw - Hill Interamericana. 2006. 10 pp.

23 Organización Panamericana de la Salud. Plan estratégico 2008 - 2012. (consultado el 22/05/2008). Disponible en http://www.paho.org/Spanish/GOV/CSP/od328-intro-s.pdf.

24 Perry, Anne Griffin. Enfermería clínica: Técnicas y procedimientos. 4ta. Edición. España. Edit. Harcourt Brace. 1999. p. 134 - 591

25 Secretaría de Salud. Modelo de Atención de Enfermería Obstétrica. México. 2005. 12 pp

\section{DIRECCIÓN PARA CORRESPONDENCIA}

Mtro. Rafael Antonio Estévez Ramos:

restevezr@hotmail.com 